\title{
An Evidence Based Review on the Current Medical Management of Allergic Rhinitis
}

\author{
Martina Munonyara ${ }^{1}$, Mohammed $\mathrm{A}^{2}$ and Tawakir Kamani ${ }^{3 *}$ \\ ${ }^{1}$ University Hospital North Staffordshire, Stoke, UK \\ ${ }^{2}$ Leicester Royal Infirmary, Leicester, UK \\ ${ }^{3}$ Leicester Royal Infirmary, Leicester, UK
}

\section{Introduction}

Allergic Rhinitis (AR) is defined as a hypersensitivity reaction of the mucous membranes in nasal cavities. It is a common condition with a prevalence of $21.8 \%$ within the United Kingdom [1]. It is categorized broadly into two groups as, Seasonal Allergic Rhinitis (SAR) and Perennial Allergic Rhinitis (PAR). SAR is associated with a particular time of the year, usually related with outdoor allergens e.g. fungi, tree, grass, and weed pollens. Whereas, PAR is associated with all year round symptoms and in particular manifests when exposed to indoor allergens such as animal proteins, dust mites, cockroaches, and moulds [2]. The symptoms of AR in particular, nasal obstruction which is associated with sleep-disordered breathing, can be bothersome leading to impairment in sleep quality and a reduction in quality of life [3]. The estimated financial burden of loss in work productivity per person annually is $€ 653$ [4]. This excludes the indirect costs associated with treatment and the social aspects of coping with AR.

$\mathrm{AR}$ is a multifactorial disease with genetic as well as environmental factors influencing disease development.Rhinitis is defined by a combination of two or more nasal symptoms: running, blocking, itching and sneezing. Allergic rhinitis occurs when inflammation occurs on exposure to allergens. This is aIgE-mediated immune response resulting from aberrant regulation by T-helper cells. Subsequent re-exposure to the allergen results in degranulation of mast cells resulting in release of arachidonic acid metabolites, chemotactic factors such as histamine, kininogenase, heparin, and other enzymes. Furthermore, inflammatory mediators, such as prostaglandin D2 and the sulfidopeptidylleukotrienes LTC4, LTD4, and LTE4, are synthesised de novo and released resulting in the acute phase response [5]. Additionally, chemo-attractants such as IL-5 are released which results in the recruitment of eosinophils, neutrophils, basophils, $\mathrm{T}$ lymphocytes, and macrophages. The $\mathrm{T}$ helper 2 lymphocytes (Th2) release IL-3, IL-4, IL-5 and other cytokines which furthers the production IgE, as well as cause eosinophil chemoattraction, eosinophil survival, and mast cell recruitment [6]. This causes an increase in capillary permeability and eosinophil infiltration which leads to the development of vascular congestion, oedema, rhinorrhoea and irritation.The development of these symptoms is known as the late phase response. Mediators also stimulate sensory nerves to cause nasal itch and congestion as well as stimulation of afferent sensory fibres which triggers sneezing [7].

Enhanced understanding of these mechanisms has helped in identifying therapeutic targets to interrupt pathways that contribute to symptoms. The complexity the pathways involved present many opportunities for the development of novel therapies however no single medicine is totally effective. The management of AR aims to control symptoms. It is divided into three components: conservative (patient education, allergen avoidance measures), pharmacotherapy and immunotherapy. We provide an updated overview of current medical management of AR.

\section{Conservative Management}

$\mathrm{AR}$ is a chronic condition which is relapsing and remitting and the consequent need for self management emphasises the importance of patient education which underpins any management strategy. This should encompass the understanding of the condition that AR presents, implications of test results and treatments. The latter should also include: benefits and risks of current therapy, techniques for administering topical medications and follow up.

Allergen avoidance and environmental control forms the hallmark of conservative therapy in AR. This involves minimising exposure to environmental trigger factors. Measures such as closing windows before bed, avoiding high pollen areas, or air filters may be used with varying efficacy. Allergen avoidance may be practical for some patients that are allergic to specific allergens such as pets and may be able to avoid these triggers. This is not as easy for others, where allergens are found in their homes, at work or the environment in which they live in and may become impractical causing more disruption to daily living. National and international guidelines of routine recommendation of house dust mite avoidance measures, particularly since these measures are expensive, time-consuming and inconvenient. Additionally, there is very little evidence of how much of the impact allergen avoidance has on quality of life and there are no recent studies which explore this.

\section{Pharmacotherapy}

A number of pharmacological medications are used in the symptomatic treatment of AR. The ARIA guidelines recommend that use of these in the treatment of AR be according to the severity and duration of AR [9]. Medications used for AR are administered nasally or orally. Intranasal medications have the advantage of delivering high concentrations of the medication directly into the nose, avoiding or minimizing systemic effects. However, intranasal distribution of medications is not optimal. Additionally, many patients with AR have conjunctivitis and/or asthma, and medications need to be administered to various targets. The agents available for treatment of AR include oral and nasal $\mathrm{H}_{1}$-Anti-histamines, intranasal corticosteroids, cromones, decongestants, intranasal anticholinergics and Leukotriene receptor antagonists. Each of these is described in detail below.

*Corresponding author: Tawakir Kamani, MD, MRCS, DOHNS, Leicester Roya Infirmary, Infirmary Close, Leicester, LE1 5WW, UK, Tel: +20129485014; Fax: +20502267016; E-mail: tawakirkamani@gmail.com

Received October 11, 2011; Accepted November 20, 2011; Published November 25, 2011

Citation: Munonyara M, Mohammed A, Kamani T (2011) An Evidence Based Review on the Current Medical Management of Allergic Rhinitis. J Aller Ther S5:003. doi:10.4172/2155-6121.S5-003

Copyright: (C) 2011 Munonyara M, et al. This is an open-access article distributed under the terms of the Creative Commons Attribution License, which permits unrestricted use, distribution, and reproduction in any medium, provided the original author and source are credited. 


\section{Oral $\mathrm{H}_{1}$-anti-histamines}

These are medications that block histamine at theH1-receptor level. Oral H1-antihistamines are effective against symptoms mediated by histamine (rhinorrhoea, sneezing, nasal itching and eye symptoms) but are less effective on nasal congestion [10]. First-generation oral H1antihistamines possess significant side effects due to their sedative and anticholinergic properties and have been superseded in use by second generation antihistamines (e.g.desloratadine, cetrizine and loratadine) which induce no [11-14] or little sedation and are not anticholinergic $[15,16]$.

Many studies have focused on the effect of oral antihistamines on SAR. A recent $\mathrm{GA}^{2} \mathrm{LEN}$ study compares the effects of desloratidine in patients with PAR: 301 patients treated with desloratidine $(5 \mathrm{mg}$ once daily) and 261 were treated with placebo. Severity scoresobtained for nasal congestion/stuffiness, sneezing, rhinorrhea/nasal discharge, nasal pruritus and eye itching were significantly reduced with desoloratidine than placebo over days 1- $85(\mathrm{P}<0.001)$ [17].

Furthermore, a trial looked at cetirizine, levocetirizine and placebo for treating childhood PAR. Seventy-four patients participated in a 12 -week treatment period. It found both cetirizine and levocetirizine lowered the mean paediatric rhinoconjunctivitis quality of life questionnaire (PRQLQ) values significantly when compared with the placebo group after the 12 -week period $(\mathrm{p}<0.05)$ [18]. These studies show the efficacy of oral antihistamines in the treatment of PAR to be effective in reducing symptoms. Oral antihistamines have the added benefit that they are safe and well tolerated in children [19].

\section{Intranasal H1-anti-histamines}

Intranasal antihistamines have been found to be effective in the managing of SAR. A randomized study comparing azelastine $0.15 \%$ and azelastine $0.10 \%$ nasal spray to a placebo in 526 subjects looked at daily total nasal symptom score (TNSS) over a period of two weeks. The results showed both azelastine $0.15 \%$ and $0.10 \%$ preparations, provided statistically significant improvements in AR symptoms as illustrated by a reduction in TNSS [20]. Also, the results were more significant in the group treated with azelastine $0.15 \%$. In addition to this, a randomized, double-blind study comparing 0.4 and $0.6 \%$ intranasal olopatadine to placebo, found that the $0.6 \%$ dose greatly improved nasal symptoms associated with AR [21].

Overall, intranasal antihistamines have a rapid onset of action which can be as quick as 30 minutes, and are well tolerated with a good safety profile. Their main side effects are irritation of nasal mucosa and bitter taste [22]. They are very effective in dealing with symptoms of nasal congestion only and do not affect other symptoms of AR.

\section{Intranasal corticosteroids}

These are generally prescribed if symptoms cannot be controlled with antihistamines. The effects of corticosteroids take several weeks to achieve best efficacy and require continuous use. Research has shown the use of nasal corticosteroids before the pollen season, achieves good symptom control [23]. Inaccurate administration technique reduces the efficacy of corticosteroid indicating that patients need to be shown the correct technique [24].

A number of randomized control trials (RCT) have looked at the ability of mometasonefuroate $200 \mathrm{mg}$ once daily versus placebo to reduce ocular symptoms epiphora, pruritus, and erythema in SAR.
There was a significant reduction intotal ocular symptom score and total nasal symptom score in these study groups when compared to placebo group $(\mathrm{P}<.001)[25,26]$.

Newer intranasal steroids have been compared in an RCT, which analysed the attributes of Fluticasone Furoate versus Fluticasone Propionate Nasal Sprays, such as: odour, immediate taste, aftertaste, and urge to taste. This RCT found a patient preference to Fluticasone Furoate $(\mathrm{P}=0.003)$ [27]. Thus, thismay be a point to consider when prescribing and where compliance is an issue.

In 2007, a Cochrane review of nasal steroids for intermittent and persistent allergic rhinitis in children found 3 RCT's which met the criteria. The review was however unable to determine which topical nasal steroid was best for children. This was mainly from the lack and poor quality data included in the trials with no reliable evidence on the effectiveness of BeclomethasoneDipropionate or flunisolide. Thus, the authors suggestedfurther research is needed on this topic [28].

With regard to efficacy, no significant difference exists between intranasal corticosteroids; however, steroids do differ in potency and their systemic absorption rates. Flunisolide, triamcinolone acetonide andbeclomethasonedipropionate have system absorption rates of 20 $50 \%$, compared to those of $\leq 0.1 \%$ for mometasonefuroate and $\leq 2 \%$ for fluticasone propionate [29].

The efficacy of intranasal steroids to reduce the symptoms of AR has been proven. Intranasal steroids reduce symptom score more than antihistamines but either can be used for first line treatment depending on the symptoms $[30,31]$.

\section{Cromones}

These are mast cell stabilizers which act by inhibiting the release of mediators in particular histamine, which result in the symptoms experienced in AR. Cromoglycate and nedocromil are available as intranasal or ocular preparations. They are modestly effective in nasal symptoms and effective in ocular symptoms [32,33,34] Cromoglycateis currently licensed in the UK for the prophylaxis of AR. They are particularly safe as they have very few side-effects, of which local irritation and transient bronchospasm are the most common. Compliance may be an issue as dosing is recommended 2-4 times a day though one preparation recommends 4-6 times a day. There is no new data on the use of sodium cromoglycate probably due to its compliance issue.

\section{Decongestants}

Decongestants, such as pseudoephedrine are used topically for nasal congestion and do not improve other symptoms associated with AR. They all contain sympathomimetic drugs which cause vasoconstriction of the mucosal blood vessels and in turn reduce oedema of the nasal mucosa. Conversely they can give rise to a rhinitis medicamentosa due to vasodilatation on withdrawal. Consequently they are not recommended for long term use of more than 10days [24].

Recent studies have focused on the combination of oral antihistamine and decongestant. A trial of a duration of 15 days looked at 598 participants with SAR, taking either desloratadine 2.5 $\mathrm{mg}$, pseudoephedrine $120 \mathrm{mg}$ or both. It found that combination treatmentwas significantly more effective than either desloratadine or pseudoephedrine mono-therapy with a reduction in TSS (total symptom score), by $-6.54(-46.0 \%)$, compared with $-5.09(-33.5 \%)$ for desloratadineand $-5.07(-35.9 \%)$ for pseudoephedrine [35]. Another trial compared combination versus mono-therapy with cetirizine and 
pseudoephedrine and found similar results with reduced the total nasal symptom score (TNSS) and visual analogue scale score compared to mono-therapy [36]. Although there is some evidence suggesting benefit of combination therapy, ARIA guidelines conditionally do not recommend this to avoid side effects from decongestants.

\section{Leukotriene receptor antagonists}

Montelukast is currently the only leukotriene receptor antagonist licensed in the United Kingdom and only licensed for patients that haveSARand concomitant asthma.

The efficacy of montelukast in the treatment of AR has been studied a number of times in the past. Considering montelukast as a monotherapy, symptom relief is not better when compared to intranasal corticosteroids [37].

Furthermore, a double-blind RCT looked at the combination of oral antihistamine (fexofenadine) andmontelukast. Three groups of patients with SAR were randomized to treatment group 1; fexofenadine; group 2 fexofenadine and montelukast; group 3 fexofenadine andplacebo. The study measured baseline changes at day 1 and at day 21. It looked at physical findings (Inferior turbinate color, oedema, nasal discharge, and congestion) and nasal resistance. It found significant difference in the results of group 2 when compared to groups 1 and 3 [38].

In studies carried out on patients with SAR and asthma, montelukast was found to improve nasal and bronchial symptoms (ARIA 1588, 1589) as well as reduce the need for use of B-agonists (puffs/day). Studies on efficacy of montelukast in PAR have not been conclusive with contradictory results $[39,40]$. Leukotriene receptor antagonists are recommended as add on therapy when symptoms are not controlled by antihistamines, intranasal steroids or a combination of both [24].

\section{Anticholinergic agents}

Anticholinergic medication is recommended for rhinorrhoea associated with AR and non-allergic rhinitis. Intranasal ipratropium bromide is administered 2-3 times daily and Topical side effects, due to the anticholinergic action, are uncommon and obviously dosedependent in their severity [41]. The most commonly experienced side effects are epistaxis, nasal dryness, and irritation. Double-blind, placebo-controlled studies have shown that ipratropium bromide is effective in controlling watery nasal discharge, but that it does not affect sneezing or nasal obstruction in PAR and non-allergic rhinitis $[42,43,44]$. There is no new data published with regard to treatment with anticholinergics.

\section{Alternative therapy}

Acupuncture is widely used by patients with AR; however there is very little evidence of the efficacy of acupuncture for treating AR. A recent systematic review which pooled the data from twelve RCTs incorporated patients with either SAR or PAR. The authors concluded that there was mixed evidence for use of acupuncture for treatment or prevention of AR. This was as a result of the small number of RCTs and small sample size [45]. Likewise, another systematic review included seven RCT's which looked at a number of outcomes: Serum IgE, medication use and symptom severity on a visual analogue scale. Although the results may have been confoundedby low patient numbers andvariable reporting of outcomes, there was insufficient evidence to prove or disprove the use of acupuncture in patients with AR.A large well conducted RCT, which overcomes the identified methodological problems are required [46]. Both systematic reviews found there was little evidence to support the use of acupuncture.

\section{Phototherapy}

A relatively new treatment isphototherapy which is used three times a day for 4.5 minutes sessions. Once symptoms are relieved, the number of treatment sessions and use can be reduced as needed. It is available over the counter. The technology neither causes heat or pain on the treated surface and it does not have any harmful effect on tissues, because the amount of UV-A and UV-B light emitted is low. The therapeutic effects of this intervention are: it inhibits antigen derived histamine release from mast cells, induces apoptosis in T-lymphocytes and eosinophil cells, reduces the number of eosinophil cells, ECP and IL-5 levels in the treated patient's nasal excretion [47].

In one study 79 patients were selected, 41 received the UVAB phototherapy and the other group of 38 patients received VIS (lowintensity visible light). Each intranasal cavity was irradiated three times a week for 2 weeks with increasing doses. No rescue medication was allowed. TNSS was recorded before commencing the trial and one month after the trial. Both groups noted a reduction in TNSS however this was more so in the active treatment group, which noted a statistically significant change $(\mathrm{p}<0.001)[48]$

In another trial, patients with SAR were treated with either intranasal phototherapy or the antihistamine fexofenadine HCL $180 \mathrm{mg}$. Eighteen patients were randomly selected to receive intranasal phototherapy and 13 received fexofenadine HCL. TNS was measured on day 1, 7 and 14 for both groups. Results showed TNS was significantly decreased in the rhinophototherapy group $4.6 \pm 2.9(\mathrm{P}<0.0001)$, but no significant difference was recorded in the fexofenadine HCL group $6.4 \pm 3.2$ after 2 weeks of treatment [49]. Though a small study it illustrates possible benefits of phototherapy as adjunct to conventional therapy of AR.

\section{Allergen immunotherapy}

Allergen specific immunotherapy (SIT) is: "the practice of administering gradually increasing quantities of an allergen product to an allergic subject to ameliorate the symptoms associated with the subsequent exposure to the causative allergen." SIT also has long term efficacy, stimulates clinical and immunologic tolerance, prevents further progression of allergic disease and improves patient quality of life. Previous terminology used to describe SIT includes desensitisation, hyposensitisation and allergy vaccination [50].

Historically, the principles of SIT described for allergic rhinitis date back to 100 years ago. Noon demonstrated that the symptoms of patients with hayfever improved after prophylactic inoculation with grass pollen [51]. Since then numerous studies, meta-analyses and reviews have studied the clinical efficacy i.e. the reduction of symptoms and the reduction of rescue medication in allergic rhinitis. More so, guidelines and recommendations on SIT as a treatment for allergic rhinitis have been complied by various worldwide societies and organisations. These include the World Health Organisation (WHO), World Allergy Organisation (WAO), Allergic Rhinitis and its Impact in Asthma (ARIA), European Academy of Allergy and Clinical Immunology (EAACI) and the British Society of Allergy and Clinical Immunology (BSCAI).

\section{Mode of mechanisms}

Apart from allergen avoidance, SIT is the only treatment against allergic disease which affects the natural course of the disease [52]. SIT is an effective treatment in both adults and children with severe allergic rhinitis who do not show a response to pharmacotherapy or allergen avoidance [55]. In particular, if the range of allergens involved 
are narrow [54]. Pharmacotherapy such as corticosteroids and antihistamines differ as they confer suppression of the symptoms. This is the converse for SIT as it targets the immune system and thus is thought to represent a cure for allergic rhinitis [55].

The mechanism of immunotherapy is complex in which SIT stimulates changes in the T-cell and antibody responses [54]. This is by altering the existing and inappropriate immune response associated with the Th2 cytokine production (i.e. IgE production and the activation and release of mast cell, eosinophils, basophils) towards the favour of Th1. In addition, T regulatory cells (Treg cells) which inhibit Th1 and Th2 cells are induced. Atopic patients show allergen-specific Th2 $\mathrm{CD}^{+}$cells, whereas healthy people show Treg responses when exposed to allergens. Thus, the purpose is to restore responses from the Th2 to Treg [55]. Antibody responses which are a feature of SIT include the development of allergen specific IgA, IgG1 and IgG4 blocking antibodies and an increased IgG4:IgE ratio. IgG4 prevents allergen-IgE complexes to B-cells and thus presentation to T-cells [52].

\section{Indications and contra-indications to SIT}

The indications for SIT in allergic rhinitis include [53]:

1. IgE-mediated seasonal pollen induced rhinitis, if symptoms have not responded adequately to optimal pharmacotherapy.

2. Selective patients with animal dander or house dust mite $(\mathrm{HDM})$ allergy in whom rigorous allergen avoidance and reasonable pharmacotherapy fail to control symptoms.

Like all treatments, the efficacy of immunotherapy depends on the appropriate selection of the patient, weighing up the benefits and risks [24]. According to BSACI, contra-indications to SIT include patients on B-blockers. Relative contraindications include chronic disorders, immunodeficiency, malignancy, autoimmune disease, patients with immunosuppressive agents and pregnancy. Uncontrolled asthma is a relative contraindication for patients with seasonal asthma due to pollens respond well to SIT. However, SIT is not recommended for uncontrolled perennial asthma [53].

Currently, SIT is mostly available as subcutaneous or sublingual immunotherapy. Indications for the use of both SCIT and SLIT are shown in (Table 1) [8].

\section{Subcutaneous immunotherapy}

Subcutaneous immunotherapy (SCIT) requires delivering a long-

\begin{tabular}{|l|l|}
\hline $\begin{array}{l}\text { Indications for subcutaneous } \\
\text { immunotherapy }\end{array}$ & $\begin{array}{l}\text { Indications for sublingual } \\
\text { immunotherapy }\end{array}$ \\
\hline $\begin{array}{l}\text { Patients with symptoms induced } \\
\text { predominantly by allergen exposure }\end{array}$ & $\begin{array}{l}\text { Carefully selected patients with rhinitis, } \\
\text { conjunctivitis and/or asthma caused by } \\
\text { pollen and mite allergy }\end{array}$ \\
\hline $\begin{array}{l}\text { Patients with a prolonged season or with } \\
\text { symptoms induced by succeeding pollen } \\
\text { seasons }\end{array}$ & $\begin{array}{l}\text { Patients insufficiently controlled by } \\
\text { conventional pharmacotherapy }\end{array}$ \\
\hline $\begin{array}{l}\text { Patients with rhinitis and symptoms } \\
\text { from lower airways during peak allergen } \\
\text { exposure }\end{array}$ & $\begin{array}{l}\text { Patients who have presented with } \\
\text { systemic reactions during injection- } \\
\text { specific immunotherapy }\end{array}$ \\
\hline $\begin{array}{l}\text { Patients in whom antihistamines and } \\
\text { moderate dose topical glucocorticoids } \\
\text { insufficiency control symptoms }\end{array}$ & $\begin{array}{l}\text { Patients showing poor compliance with } \\
\text { or refusing injections }\end{array}$ \\
\hline $\begin{array}{l}\text { Patients who do not want to be on } \\
\text { constant or long-term pharmacotherapy }\end{array}$ & $\begin{array}{l}\text { Patients in whom pharmacotherapy } \\
\text { induces undesirable side-effects }\end{array}$ \\
\hline
\end{tabular}

Table 1: Indications for SCIT and SLIT (taken from ARIA guidelines 2008) term course of repeated deep subcutaneous injections with extracts of allergen. Currently, licensed in the UK for the treatment of allergic rhinitis for grass or tree pollen is Pollinex ${ }^{\bullet}$. Other vaccines which are unlicensed are also used [53]. The SCIT regime usually involves an updosing phase of regular weekly injections for $8-16$ weeks. At this point, patients are started with a very low dose of allergen increased with gradual increments until a maintenance dose is achieved. Subsequently, the patient undergoes monthly maintenance injections for a period o f 3-5 years at 4-6 week intervals [54,57]. Most SCIT injections contain a standardised dose of $5-20$ micrograms which is thought to be the optimal dose [58].

\section{Efficacy of SCIT}

Clinical efficacy has been identified for specific allergens such as grass, birch, ragweed, Russian thistle, Parietaria pollen, mites and cat [59]. In 2007, the most recent Cochrane review was published on SCIT for SAR. This review involved 2871 patients in 51 trials with SAR due to tree, grass or weed pollens noted a significant reduction in symptoms and medication scores use with a relatively low risk of severe adverse events. Thus concluding SCIT is an effective treatment for allergic rhinitis [60].

The clinical efficacy of SCIT for perennial rhinitis is reported to be of less clinical benefit compared to that for SAR [54]. The clinical benefit of SCIT for house dust mite was concluded as uncertain and other allergens very uncertain. Evidence is low for the role of SCIT in perennial rhinitis compared to that for SAR [61]. This is thought to be a reflection in the difficultly in determining the extent to which is allergy is responsible for perennial symptoms [54]

\section{Safety}

Since this treatment requires exposing the patient to an allergen, the potential adverse reactions are a limitation to this treatment Adverse reactions to SCIT can be classified into two groups: either local i.e. pruritis, urticaria, erythema or swelling over the site of injection; or as systemic i.e. mild to severe anaphylaxis. There have been reports of fatalities associated to SCIT, the majority of which are in patients who had co-existing asthma which is poorly controlled. Thus, asthma has been suggested as a contributing factor. Similarly, near-fatal experiences have mostly involved asthmatics [62]. SCIT is therefore administrated by medical staff in allergy clinics trained in the management of anaphylaxis with full access to resuscitation facilities [55]. Patients are supervised for 60 minutes after administration of SCIT [57].

Historically, discrepancies of when to treat anaphylaxis between various authors made it difficult to know when it was best to treat. In 2010 a collaborative including the WAO formed a consensus on SCITinduced systemic reactions (SR) grading reactions from Grade 1 to 5 providing clinical guidance on assessing when to treat the SR with adrenaline. Additionally, this aids grading of reactions in research, allowing surveillance and comparisons to be made between different clinical trials [63].

\section{Sublingual immunotherapy}

Non-injection or local routes such as nasal, oral and bronchial routes have been explored as safer routes of administration, as SCIT is limited by its adverse effects [64]. Sublingual immunotherapy remains as the most likely to be acceptable and effective local route [65] Currently, SLIT is used more frequently than SCIT in some European countries due to efficacy, better safety, better patient compliance and cost-effectiveness [66]. SLIT is administered either as a tablet or in 
drops under the tongue for 1-2 minutes and then swallowed, commonly known as sublingual-swallow route [62].

\section{Efficacy}

An increase in number of studies on SLIT has resulted in recent improvements in better trial design. The recent Cochrane review on SLIT looked at 49 trials with 2333 SLIT and 2256 placebo patients. The meta-analysis reinforced that SLIT causes significant reduction in symptom and medication scores compared to placebo. The optimum dose and duration of therapy is however still unknown [67].

SLIT is seen effective for grass allergens in SAR. ARIA concluded the effect on nasal symptoms is probably small to moderate when SLIT is used for pollen but still may be of clinical benefit [61]. A recent meta-analysis in 2010 concluded SLIT for grass allergens is effective in patients with SAR compared to placebo with significant reduction in symptoms and medication use. An increased response rate was seen to be best achieved with the pre-seasonal use of SLIT for 12 weeks or greater. A course of treatment for 12 months was most beneficial with treatment continued more than 12 months producing lower symptom scores [68]. The Cochrane review states that, although further studies are required, there does not appear to be a greater effect of SLIT with perennial allergens when compared to seasonal allergens $[53,67]$.

\section{Safety}

The safety prolife of SLIT is better compared to that of SCIT with few documented cases of anaphylaxis and no known fatalities [67]. Although rare, the most commonly described include easily managed mild reactions such as buccal or labial itching, oedema and gastrointestinal effects such as vomiting; which tend to occur in studies involving higher doses $[59,67,69]$.

SLIT can be self-administrated by patients within the home setting. Although, SR are deemed rare there is the potential consequence that a SR can be serious if taken by a patient at home. Therefore, it is recommended for the safety of the patient to be advised on what to do if a SR should occur [53,59].

\section{SCIT Vs SLIT}

Although comparative studies exist; Radulovic and colleagues stated in the recent Cochrane review there was insufficient data to conclude between the two therapies and more trials comparing are required [67]. Compared to SCIT, SLIT proved to be more costeffective in a European study conducted over 3 years from the point of view of the patient, society and third-party payer. The study showed 3-year SIT expenditures per patient to be $€ 684$ vs $€ 1,004$ for SLIT and SCIT respectively [70].

\section{Conclusion}

$\mathrm{AR}$ is a disease which contributes to significant morbidity and reduction in quality of life to its sufferers. Therefore, adequate control of symptoms of AR is important to ameliorate the quality of life, as well as improving asthma control in patients with concomitant asthma.

A plethora of treatments for the medical management of AR exist for the physician, which have been reinforced by various guidelines and systematic reviews. However, much research is still required in various areas of its medical management.

$\mathrm{AR}$ requires much self management, so patient education is important. The balance of the benefits and the risks of each of the available treatments need to be considered in each individual case.
Pharmacotherapy is safe and has a robust evidence base in the indication of both SAR and PAR. However, a combination of therapies may be required to obtain adequate symptom relief.

Alternative therapies such as acupuncture have a weak evidence base, thus requiring further research as a treatment. Similarly, the use of phototherapy requires further research but is a potential adjunct to pharmacotherapy.

Immunotherapy is becoming more acceptable due to the increasing evidence base available. However, there is less evidence to supports its indications for PAR and it is still flawed by its safety due to the risks of anaphylaxis.

Understanding the mechanisms behind AR has been integral inidentifying therapeutic targets to interrupt the pathways contributing to the symptoms. More so, the complexity the pathways involve present many opportunities for the development of novel therapies as no single medicine is totally effective.

\section{References}

1. Canonica GW, Bousquet J, Mullol J, Scadding GK, Virchow JC (2007) A survey of the burden of allergic rhinitis in Europe. Allergy 62: 17-25.

2. Singh AB, Kumar $P$ (2003) Aeroallergens in clinical practice of allergy in India An overview. Ann Agric Environ Med 10:131-136.

3. Nathan RA (2007) The burden of allergic rhinitis. Allergy Asthma Proc 28: 3-9.

4. Hellgren J, Cervin A, Nordling S, Bergman A, Cardell LO (2010) Allergic rhinitis and the common cold--high cost to society. Allergy 65: 776-783.

5. Al Suleimani YM, Walker MJ (2007) Allergic rhinitis and its pharmacology. PharmacolTher 114: 233-260.

6. Hansen I, Klimek L, Mosges R, Hormann K (2004) Mediators of inflammation in the early and the late phase of allergic rhinitis. Current Opinion in Allergy \& Clinical Immunology 4: 159-163.

7. Pfaar O, Raap U, Holz M, Hormann K, Klimek L (2009) Pathophysiology of itching and sneezing in allergic rhinitis. Swiss Med Wkly 139: 35-40.

8. Bousquet J, Khaltaev N, Cruz AA, Denburg J, Fokkens WJ, et al. (2008) Allergic Rhinitis and its Impact on Asthma (ARIA) 2008 Update (in collaboration with the World Health Organization, GA2LEN and AllerGen). Allergy: 63: 8-160.

9. Simons FE (2004) Advances in H1-antihistamines. N Engl J Med 351: 2203 2217.

10. Simons FE, PrennerBM, Finn A Jr (2003) Efficacy and safety of desloratadine in the treatment of perennial allergic rhinitis. J Allergy Clin Immunol 111: 617-622.

11. Howarth PH, Stern MA, RoiL, Reynolds R, BousquetJ (1999) Doubleblind placebo-controlled study comparing the efficacy and safety of fexofenadine hydrochloride (120 and $180 \mathrm{mg}$ once daily) and cetirizine in seasonal allergic rhinitis. J Allergy ClinImmunol104: 927-933.

12. Skassa-BrociekW, BousquetJ, Montes F, VerdierM, Schwab D, et al. (1988) Double-blind placebo-controlled study of loratadine, mequitazine, and placebo in the symptomatic treatment of seasonal allergic rhinitis. J Allergy Clinlmmunol 81: $725-730$.

13. Hindmarchl, ShamsiZ (2001) The effects of single and repeated administration of ebastine on cognition and psychomotor performance in comparison to triprolidine and placebo in healthy volunteers. Curr Med Res Opin 17: 273-281.

14. PradalierA, NeukirchC, Dreyfus I, DevillierP (2007) Desloratadine improves quality of life and symptom severity in patients with allergic rhinitis. Allergy 62 1331-1334.

15. BachertC, BousquetJ, CanonicaGW, Durham SR, KlimekL, et al. (2004) Levocetirizine improves quality of life and reduces costs in long-term management of persistent allergic rhinitis. J Allergy ClinImmunol 114: 838-844.

16. Berman BA (1990) Perennial allergic rhinitis:clinical efficacy of a new antihistamine.J Allergy Clin Immunol 86: 1004-1008. 
Citation: Munonyara M, Mohammed A, Kamani T (2011) An Evidence Based Review on the Current Medical Management of Allergic Rhinitis. J Aller Ther S5:003. doi:10.4172/2155-6121.S5-003

17. Bousquet J, Bachert C, Canonica GW, Mullol J, Van Cauwenberge $P$, et al (2010) Efficacy of desloratadine in persistent allergic rhinitis - a GA(2)LEN study. Int Arch Allergy Immunol 153: 395-402.

18. CF, Sun HL, Lu KH, Ku MS, Lue KH (2009) The comparison of cetirizine, levocetirizine and placebo for the treatment of childhood perennial allergic rhinitis. Pediatr Allergy Immunol 20: 493-499.

19. Phan H, Moeller ML, Nahata MC (2009) Treatment of Allergic Rhinitis in Infants and Children Efficacy and Safety of Second-Generation Antihistamines and the Leukotriene Receptor Antagonist Montelukast. Drugs 69: 2541-2576.

20. Shah S, Berger W, Lumry W, La Force C, Wheeler W, et al. (2009) Efficacy and safety of azelastine $0.15 \%$ nasal spray and azelastine $0.10 \%$ nasal spray in patients with seasonal allergic rhinitis. Allergy \& Asthma Proceedings 30: 628-633.

21. Roland PS, Marple BF, Wall GM (2010) Olopatadine nasal spray for the treatment of allergic rhinitis. Expert Rev ClinImmunol 6: 197-204.

22. BE, Harder JM (2009) Antihistamine treatment for allergic rhinitis: different routes, different outcomes? Allergy Asthma Proc 30: 589-594

23. Laekeman G, Simoens S, Buffels J, Gillard M, Robillard T, et al. (2010) Continuous versus on-demand pharmacotherapy of allergic rhinitis: evidence and practice. Respir Med 104: 615-625.

24. Scadding GK, Durham SR, Mirakian R, Jones NS, Leech SC, et al. (2008) $\mathrm{BSACl}$ guidelines for the management of allergic and non-allergic rhinitis. ClinExp Allergy 38: 19-42.

25. Prenner BM, Lanier BQ, Bernstein DI, Shekar T, Teper A (2010) Mometasonefuroate nasal spray reduces the ocular symptoms of seasonal allergic rhinitis. J Allergy Clin Immunol 125: 1247-1253.

26. Bielory L (2008) Ocular symptom reduction in patients with seasonal allergic rhinitis treated with the intranasal corticosteroid mometasonefuroate. Annals of Allergy, Asthma, \& Immunology 100: 272-279.

27. Meltzer EO, Stahlman JE, Leflein J, Meltzer S, Lim J, et al. (2008) Preferences of adult patients with allergic rhinitis for the sensory attributes of fluticasone furoate versus fluticasone propionate nasal sprays: a randomized, multicenter, double-blind, single-dose, crossover study. ClinTher 30: 271-279.

28. Al Sayyad JJ, Fedorowicz Z, Alhashimi D, Jamal A (2007) Topical nasal steroids for intermittent and persistent allergic rhinitis in children. Cochrane Database Syst Rev 24: CD003163.

29. Min YG (2010) The pathophysiology, diagnosis and treatment of allergic rhinitis. Allergy, Asthma and Immunology Research 2: 65-76.

30. Carr WW (2008) Pediatric allergic rhinitis: current and future state of the art Allergy Asthma Proc 29: 14-23.

31. Georgalas C, Terreehorst I, Fokkens W (2010) Current management of allergic rhinitis in children. Pediatric Allergy \& Immunology 21: 119-126.

32. Meltzer EO (2002) Efficacy and patient satisfaction with cromolyn sodium nasal solution in the treatment of seasonal allergic rhinitis: a placebo controlled study. ClinTher 24: 942-952.

33. SchullerDE, SelcowJE, JoosTH, HannawayPJ, Hirsch SR, et al. (1990) A multicenter trial of nedocromil sodium, $1 \%$ nasal solution, compared with cromolyn sodium and placebo in ragweed seasonal allergic rhinitis. J Allergy Clinlmmunol 86: 554-561.

34. James IG, Campbell LM, Harrison JM, Fell PJ, Ellers-Lenz B, et al. (2003) Comparison of the efficacy and tolerability of topically administered azelastine, sodium cromoglycate and placebo in the treatment of seasonal allergic conjunctivitis and rhino-conjunctivitis. Curr Med Res Opin 19: 313-320.

35. Grubbe RE, Lumry WR, Anolik R (2009) Efficacy and safety of desloratadine/ pseudoephedrine combination vs its components in seasonal allergic rhinitis. $J$ InvestigAllergolClinlmmunol 19: 117-124.

36. Badorrek P, Dick M, Schauerte A, Hecker H, Murdoch R, et al. (2009) A combination of cetirizine and pseudoephedrine has therapeutic benefits when compared to single drug treatment in allergic rhinitis. Int J Clin Pharmacol Ther 47: 71-77.

37. Lagos JA, Marshall GD (2007) Montelukast in the management of allergic rhinitis. Ther Clin Risk Manag 3: 327-332.

38. Cingi C, Gunhan K, Gage-White L, Unlu H (2010) Efficacy of leukotriene antagonists as concomitant therapy in allergic rhinitis. Laryngoscope 120: 1718-1723.
39. Patel P, Philip G, Yang W, Call R, HorakF, et al. (2005) Randomized, doubleblind, placebo-controlled study of montelukast for treating perennial allergic rhinitis. Ann Allergy Asthma Immunol 95: 551-557.

40. Philip G, Williams-Herman D, Patel P, Weinstein SF, AlonA, et al. (2007) Efficacy of montelukast for treating perennial allergic rhinitis. Allergy Asthma Proc 28: 296-304.

41. BousquetJ, Van CauwenbergeP, KhaltaevN (2001) Allergic rhinitis and its impact on asthma. J Allergy Clin Immunol 108: S147-S334.

42. MygindN, BorumP (1989) Intranasal ipratropium: literature abstracts and comments. RhinolSuppl 9: 37-44.

43. BorumP, MygindN, Schultz Larsen F (1979) Intranasal ipratropium: a new treatment for perennial rhinitis. Clin Otolaryngol 4: 407-411.

44. WagenmannM, NaclerioRM (1992) Complications of sinusitis. J Allergy Clinlmmunol 90: 552-554.

45. Lee MS, Pittler MH, Shin BC, Kim JI, Ernst E (2009) Acupuncture for allergic rhinitis: a systematic review. Ann Allergy Asthma Immunol 102: 269-279.

46. Roberts J, Huissoon A, Dretzke J, Wang D, Hyde C (2008) A systematic review of the clinical effectiveness of acupuncture for allergic rhinitis. BMC Complement Altern Med 8: 13.

47. Kemeny L, Koreck A (2007) Ultraviolet light phototherapy for allergic rhinitis. PhotochemPhotobiol B 87: 58-65.

48. Cingi C, Cakli H, Yaz A, Songu M, Bal C (2010) Phototherapy for allergic rhinitis: a prospective, randomized, single-blind, placebo-controlled study. TherAdvRespir Dis 4: 209-213.

49. Garaczi E, Boros-Gyevi M, Bella Z, Csoma Z, Kemeny L, et al. (2011) Intranasa phototherapy is more effective than fexofenadine hydrochloride in the treatmen of seasonal allergic rhinitis: results of a pilot study. PhotochemPhotobiol 87: 474-477.

50. Alvarez-Cuesta E, Bousquet J, Canonica GW, Durham SR, Malling HJ, et al. (2006) Standards for practical allergen-specific immunotherapy. Allergy 61 $1-20$.

51. Noon L (1911) Prophylactic inoculations against hay fever. The Lancet 1:1572 1573.

52. Pipet A, Botturi K, Pinot D, Vervloet D, Magnan A (2009) Allergen-specific immunotherapy in allergic rhinitis and asthma. Mechanisms and proof of efficacy. Respir Med 103: 800-812.

53. Walker SM, Durham SR, Till SJ, Roberts G, Corrigan CJ, et al. (2011) Immunotherapy for allergic rhinitis. Clinical \& Experimental Allergy 41: 11771200.

54. Frew AJ (2010) Allergen immunotherapy. J Allergy Clinlmmunol 125: S306-S313.

55. Greiner AN, Hellings PW, Rotiroti G, Scadding GK (2011) Allergic rhinitis. The Lancet.

56. Moingeon P, Batard T, Fadel R, Frati F, Sieber J, et al. (2006) Immune mechanisms of allergen-specific sublingual immunotherapy. Allergy 61: 151 165

57. Allergen Immunotherapy: A synopsis (2004)

58. Bousquet J, Lockey R, Malling HJ (1998) Allergen immunotherapy: therapeutic vaccines for allergic diseases. A WHO Position Paper. J Allergy Clin Immuno 102: 58-62.

59. Bousquet J, Schunemann HJ, Zuberbier T, Bachert C, Baena-Cagnani CE, et al. (2010) Development and implementation of guidelines in allergic rhinitis - an ARIA-GA2LEN paper. Allergy 65: 1212-1221.

60. Calderon MA, Alves B, Jacobson M, Hurwitz B, Sheikh A, et al. (2007) Allergen injection immunotherapy for seasonal allergic rhinitis. Cochrane Database Syst Rev: CD001936.

61. Brozek JL, Bousquet J, Baena-Cagnani CE, Bonini S, Canonica GW, et al. (2010) Allergic Rhinitis and its Impact on Asthma (ARIA) guidelines: 2010 revision. J Allergy Clin Immunol 126: 466-476.

62. Canonica GW, Bousquet J, Casale T, Lockey R, Baena-Cagnani C, et al. (2009) Sublingual Immunotherapy: World Allergy Organization Position Paper 2009 Allergy 64: 1-59. 
Citation: Munonyara M, Mohammed A, Kamani T (2011) An Evidence Based Review on the Current Medical Management of Allergic Rhinitis. J Aller Ther S5:003. doi:10.4172/2155-6121.S5-003

Page 7 of 7

63. Cox L, Larenas-Linnemann D, Lockey RF, Passalacqua G (2010) Speaking the same language: The World Allergy Organisation Subcutaneous Immunotherapy Systemic Reaction Grading System. J Allergy Clin Immunol 125: 569-574.

64. Canonica GW, Passalacqua G (2003) Noninjection routes for immunotherapy. J Allergy Clin Immunol 111: 437-448.

65. Wilson DR, Torres-Lima M, Durham S (2005) Sublingual immunotherapy for allergic rhinitis: systematic review and meta-analysis. Allergy 60: 4-12.

66. Incorvaia C, Masieri S, Scurati S, Soffia S, Puccinelli P, et al. (2011) The current role of sublingual immunotherapy in the treatment of allergic rhinitis in adults and children. J Asthma Allergy 4: 13-17.
67. Radulovic S, Calderon MA, Wilson D, Durham S (2010) Sublingual immunotherapy for allergic rhinitis. Cochrane Database of Syst Rev: CD002893.

68. Di Bona D, Plaia A, Scafidi V, Leto-Barone MS, Di Lorenzo G (2010) Efficacy of sublingual immunotherapy with grass allergens for seasonal allergic rhinitis: a systematic review and meta-analysis. J Allergy Clin Immunol 126: 558-566.

69. Passalacqua G, Guerra L, Compalati E, Canonica GW (2007) The safety of allergen specific sublingual immunotherapy. Curr Drug Saf 2: 117-123.

70. Podladnikova J, Krcmova I, VIcek J (2008) Economic evaluation of sublingual vs subcutaneous allergen immunotherapy. Ann Allergy Asthma Immunol 100: 482-489.

This article was originally published in a special issue, Allergic Rhinosinusitis handled by Editor(s). Reginald F. Baugh, University of Toledo, USA. 